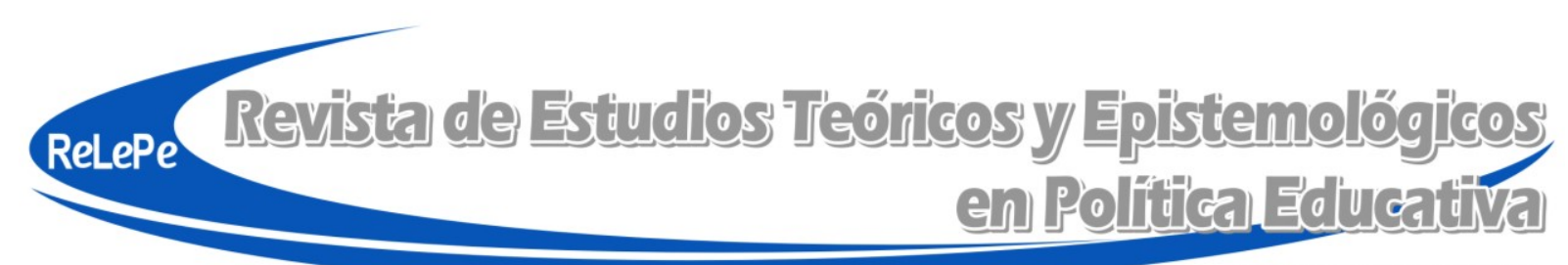

ISSN 2409-3696

DOI: $10.5212 /$ retepe.v.4.009

\title{
Implementação das Políticas Educacionais: tendências das pesquisas publicadas (2007-2017)
}

\author{
Ana Cristina Prado de Oliveira \\ Universidade Federal do Estado do Rio de Janeiro - UNIRIO \\ ana.prado.oliveira@gmail.com
}

\begin{abstract}
Resumo: O artigo apresenta um amplo levantamento de artigos acadêmicos no campo da implementação de políticas educacionais publicados no período de 2007 a 2017. O levantamento bibliográfico foi realizado em importantes periódicos da área educacional, sendo cinco nacionais e dois internacionais, resultando na seleção de 153 artigos sobre o tema. Analisar a produção acadêmica a partir das recentes pesquisas publicadas sobre a implementação de políticas educacionais parece ser importante contribuição teórica e metodológica para o campo. Através da disseminação dos estudos levantados, propõe-se uma análise dos mesmos a partir de diferentes ângulos: impacto do tema na publicação acadêmica recente, referências teóricas apontadas, metodologias de pesquisa utilizadas e categorização temática. Tomando como referência os estudos sobre a Regulação de Políticas Educacionais (BARROSO, 2006) e sobre a Burocracia de Nível de Rua (LIPSKY, 1980; LOTTA, 2015), a análise categórica dos artigos destacou, especialmente, aqueles que abordaram o papel dos diferentes agentes na implementação de políticas educacionais. A sistematização, análise e categorização destas publicações, além de importante contribuição para os estudos sobre o tema, aponta novas possibilidades de diálogo no campo.
\end{abstract}

Palavras-chave: Implementação. Políticas Educacionais. Regulação.

\section{Education policy implementation: trends of research publications (2007-2017)}

\begin{abstract}
The article presents a broad review of academic articles in the field of educational policy implementation published between 2007 and 2017. The literature review was carried out in important journals in the educational area, five were national ones and two were international ones, resulting in the selection of 153 articles on the theme. Analyzing academic production from the recent published research on the implementation of educational policies seems to be an important theoretical and methodological contribution to the field. Through the dissemination of the studies, it is proposed to analyze them from different perspectives: the impact of the subject in the recent academic publication, theoretical references pointed out, research methodologies used and thematic categorization. Taking the reference of the studies on Educational Policy Regulation (BARROSO, 2006) and on Street Level Bureaucracy (LIPSKY, 1980; LOTTA, 2015), the categorical analysis of the articles highlighted, especially, those that addressed the role of the different agents in the implementation of educational policies. The systematization, analysis, and categorization of these publications, more than an important contribution to the studies on the theme, points out new possibilities for dialogue in the field.
\end{abstract}

Keywords: Policy implementation. Education policy. Regulation. 
Implementación de politicas educativas: tendencias de investigaciones publicadas (2007-2017)

Resumen: El artículo presenta un amplio levantamiento de artículos académicos en el campo de la implementación de políticas educativas, publicados en el período de 2007 a 2017. El levantamiento bibliográfico fue realizado en importantes periódicos del área educativa, siendo cinco nacionales y dos internacionales, resultando en la selección de 153 artículos sobre el tema. Analizar la producción académica a partir de las recientes investigaciones publicadas sobre la implementación de políticas educativas parece ser importante contribución teórica y metodológica para el campo. A través de la diseminación de los estudios levantados, se propone un análisis de estos a partir de diferentes ángulos: impacto del tema en la publicación académica reciente, referencias teóricas apuntadas, metodologías de investigación utilizadas y categorización temática. El análisis categórico de los artículos destacó, especialmente, aquellos que abordaron el papel de los diferentes agentes en la implementación de políticas educativas, tomando como referencia los estudios sobre la Regulación de la Política Educacional (BARROSO, 2006) Burocracia de Nivel de Calle (LIPSKY, 1980; LOTTA, 2015). La sistematización, análisis y categorización de estas publicaciones, además de una importante contribución a los estudios sobre el tema, apunta nuevas posibilidades de diálogo en el campo.

Palabras clave: Implementación. Políticas educativas. Regulación.

\section{Introdução}

O artigo em tela apresenta um amplo levantamento de artigos acadêmicos no campo da implementação de políticas educacionais publicados no período de 2007 a $2017^{1}$. Este levantamento integra um projeto de pesquisa em andamento que propõe uma análise sobre a atuação de diferentes agentes implementadores de uma política educacional (Turno Único Carioca) no município do Rio de Janeiro. Entendemos que o estudo sobre as Políticas Públicas em Educação em suas diferentes abordagens torna-se, cada vez mais, essencial para a compreensão dos processos educacionais e escolares, especialmente quando se pretende compreender as dinâmicas de adaptação desenvolvidas a partir das proposições de uma agenda política que, nem sempre, considera as especificidades de seu campo de abrangência. Neste sentido, analisar a produção acadêmica a partir das recentes pesquisas publicadas sobre o tema, parece ser importante contribuição teórica e metodológica, através da disseminação dos estudos levantados, propondo uma análise dos mesmos a partir de diferentes ângulos: impacto do tema na publicação acadêmica recente, referências teóricas apontadas, metodologias de pesquisa utilizadas e categorização temática. Nosso objetivo neste levantamento foi analisar a relevância do tema no campo, aprofundando e atualizando os estudos sobre a implementação/recontextualização das políticas educacionais nos espaços escolares. Acreditamos que a sistematização, análise e categorização destas publicações, além de importante contribuição para os estudos sobre o tema, aponta novas possibilidades de diálogo no campo. Este artigo apresenta o resultado analítico deste levantamento e as seções que se seguem descrevem este percurso, iniciando por uma breve sistematização de nossa compreensão acerca dos estudos sobre implementação de políticas públicas na educação.

\footnotetext{
1 Este levantamento bibliográfico contou com a preciosa contribuição dos bolsistas de Iniciação Científica Igor Rodrigues Gandra, Isabelle Premoli Parada e Millena Lopes Rocha.
} 


\section{Os estudos sobre as políticas educacionais: o papel dos implementadores}

Um discurso, como o veiculado por uma política pública educacional, pode estar sujeito a várias possibilidades de recontextualização, nos diferentes contextos, e através dos diferentes atores envolvidos. "O conceito de recontextualização permite a análise da emergência e desenvolvimento dos discursos de políticas específicas ao longo do tempo" (MAINARDES; STREMEL, 2010, p. 46). Por meio da recontextualização, o discurso se desloca do seu contexto original de produção para outro contexto onde é modificado (através de seleção, simplificação, condensação e reelaboração) e relacionado com outros discursos, sendo depois realocado. (MAINARDES; STREMEL, 2010, p. 43).

Assim, o discurso original de uma política passa por uma transformação, criando o discurso recontextualizado. Esse processo de transformação envolve releituras, reinterpretações, mudanças de significados. Conforme aponta Barroso (2006, p. 171) as escolas contam com suas lógicas de regulação interna, que se diferenciam entre si dependendo do seu contexto. Por regulação interna entendemos os processos formais e informais que garantem a coordenação da ação coletiva na escola, através da produção e manutenção de regras que asseguram seu funcionamento. Para o autor (2006, p. 189), “a escola constitui um espaço central do processo de regulação das políticas educativas mediatizando e transformando os efeitos da regulação institucional externa (central e intermediária)".

Tradicionalmente, os estudos sobre implementação de políticas tendiam "a focalizar os processos de tomada de decisão, assumindo a implementação como um processo hierárquico que vinha de cima para baixo" (LOTTA, 2015, p. 34), em uma perspectiva analítica que ficou conhecida como policy-centered. Considera-se, nesta perspectiva, que toda a implementação de uma política pode ser controlada e seus resultados são previsíveis. Quando os resultados alcançados passaram a ser analisados e apresentaram dissonância dos resultados previstos, passou-se a considerar que esta poderia ser uma consequência de diferentes formas de implementação (diferentes interpretações, diferentes motivações, autonomia dos implementadores). No que poderia ser considerado como uma segunda geração de estudos sobre a implementação, em uma perspectiva da negociação, valoriza-se a política pública em ação. A discricionariedade dos implementadores passa a ser focalizada, em um movimento de olhar a implementação como um conjunto de tensões, interações e estratégias que envolvem as tomadas de decisão. De acordo com Lotta (2015), simultaneamente a este movimento, surgem "alguns estudos sobre os agentes de rua, que exploravam a existência e a natureza da discricionariedade nos ambientes organizacionais, sendo o principal deles a Street Level Burocracy, de Lipsky". Na década de 1980, diferentes campos de estudo divergiam na análise da implementação de políticas públicas, sendo considerados essencialmente prescritivos (top-down) ou descritivos (botton-up). Nestas duas abordagens na análise da implementação das políticas públicas há um distanciamento entre os espaços de formulação e de execução, separando administradores e executores.

Mais recentemente (a partir dos anos de 1990), os novos modelos de Estado trazem a consideração sobre um processo dinâmico e interativo na implementação das políticas públicas. Neste sentido, vários estudos buscaram sintetizar as contribuições dos modelos de análise anteriores entendendo o processo decisório na implementação como algo central e contínuo. Neste campo múltiplo, o elemento base de análise passa a ser a discricionariedade dos agentes implementadores. De acordo com Lotta (2015, p. 40) esta tomada de decisão significa considerar que "as políticas são realizadas com base na interação de diversos atores que transformam os processos e, assim, parte da análise da dinâmica interativa que há por trás das políticas públicas”. A literatura que se dedica à análise de políticas públicas, sobretudo aquela que se debruça sobre os processos de sua implementação, trata os agentes implementadores na categoria de burocratas. 
Neste campo, a Street Level Burocracy, proposta por Lipsky, tem destaque ao se dedicar ao estudo dos burocratas de nível de rua, aqueles que "trabalham diretamente no contato com os usuários dos serviços públicos" (LOTTA, 2015, p. 43). Nesta perspectiva, os agentes implementadores deixam de apenas executar e passam a participar dos processos políticos através de escolhas e negociações, além de serem intermediários entre o governo e os cidadãos. Estes atuam não apenas executando ações predeterminadas, mas influenciam os resultados da implementação através de seu espaço de discricionariedade, motivados por diversos fatores que impactam suas escolhas e atuação. "São esses agentes que determinam o acesso do público a direitos e benefícios governamentais e é por meio deles que a população consegue acessar a administração pública" (LOTTA, 2015, p. 43). Partindo desta perspectiva analítica, para compreender a implementação e os efeitos de uma política, torna-se necessário considerar este espaço de discricionariedade dos implementadores nos diferentes níveis em que ocorre a implementação.

Tendo como referência os estudos sobre a implementação de políticas públicas, aqui brevemente sumarizados, a revisão bibliográfica em tela pretende identificar, em sua categorização temática, quais são as perspectivas analíticas adotadas nestes estudos no campo educacional. Busca-se, ainda, discutir se e em que medida os artigos acadêmicos publicados sobre o tema consideram o papel dos agentes implementadores em suas análises.

\section{Escolhas metodológicas: a seleção dos artigos}

A revisão bibliográfica proposta por este trabalho pode ser considerada uma revisão sistemática de cunho exploratório. Revisão sistemática, pois, pretende apresentar uma síntese das publicações (artigos acadêmicos) sobre a temática de interesse, buscando apontar as tendências teóricas e metodológicas dos estudos recentes no campo, bem como identificar potencialidades e lacunas neste levantamento. De acordo com Davies (2007, p. 32),

As revisões sistemáticas são uma forma de síntese de pesquisas que contribui para as políticas e práticas baseadas em evidências ao identificar as evidências de pesquisa acumuladas sobre um tópico ou uma questão, avaliando-as criticamente em relação a sua metodologia e suas conclusões e determinando as mensagens coerentes e variáveis que são geradas por esse corpus de trabalho. As revisões sistemáticas das evidências de pesquisa existentes também ajudam a identificar o que se conhece sobre um tópico ou questão e, assim, a direcionar novas pesquisas primárias nas áreas em que haja uma lacuna na base de evidências.

E trata-se de um trabalho de cunho exploratório pois não pretende realizar uma análise profunda ou comparada dos resultados encontrados pelos estudos analisados nem tampouco uma meta-análise a partir de suas conclusões. Sua intenção é delimitar, a partir de seu recorte, a produção científica sobre a implementação de políticas educacionais identificando suas principais referências teóricas, as tendências metodológicas e suas contribuições para o campo. Especialmente, procurou localizar aqueles estudos que se aproximam do desenho do projeto de pesquisa que originou este levantamento, enfocando os diferentes espaços de ação dos agentes na implementação das políticas educacionais.

O levantamento bibliográfico foi realizado em periódicos da área educacional, sendo cinco nacionais e dois internacionais. A seleção dos periódicos procurou considerar aqueles de temática ampla e variada (Revista Brasileira de Educação, Educação e Sociedade, Cadernos de Pesquisa e American Educational Research Journal) e outros com a temática mais específica, direcionada ao nosso tema de interesse (Jornal de Políticas Educacionais, Revista Brasileira de Política e Administração da Educação e Educational Administration Quarterly). Nesta escolha, 
optamos por priorizar periódicos vinculados a associações/núcleos de pesquisa (Revista Brasileira de Educação/ANPED; Educação e Sociedade/CEDES; Cadernos de Pesquisa/FCC; American Educational Research Journal/AERA; Revista Brasileira de Política e Administração da Educação /ANPAE; Jornal de Políticas Educacionais/NUPE-UFPR; Educational Administration Quarterly/UCEA) ${ }^{2}$ além de considerar os aspectos relacionados ao seu impacto no campo (disponibilidade nas plataformas de pesquisa e escolha por pesquisadores do campo para publicação e/ou citação).

O recorte temporal assumiu o período de 2007 a 2017 pretendendo um olhar ampliado sobre a produção temática recente. A seleção dos artigos foi realizada a partir da leitura dos sumários, com uma pré-seleção a partir dos títulos de todos os 2530 artigos publicados no período. Em seguida, consultava-se os resumos para validação da seleção, levando em consideração a apresentação da temática pesquisada pelos autores de cada artigo pré-selecionado. Selecionamos aqueles artigos com abordagem explícita do tema implementação de políticas educacionais na educação básica. Foram selecionados 153 artigos e sua distribuição entre os periódicos é apresentada no quadro que se segue.

Quadro 1 - Levantamento Bibliográfico - Distribuição

\begin{tabular}{|c|c|c|c|c|c|}
\hline Periódico & Período & $\begin{array}{l}\text { Volumes } \\
\text { consulta- } \\
\text { dos }\end{array}$ & $\begin{array}{l}\text { Números } \\
\text { consultados }\end{array}$ & $\begin{array}{c}\text { Artigos } \\
\text { publicados } \\
\text { no período }\end{array}$ & $\begin{array}{l}\text { Artigos } \\
\text { selecionados }\end{array}$ \\
\hline Revista Brasileira de Educação & $\begin{array}{c}11 \text { anos } \\
(2007-2017)\end{array}$ & 11 & 36 & 437 & $\begin{array}{c}14 \\
(3,2 \%)\end{array}$ \\
\hline Educação e Sociedade & $\begin{array}{c}11 \text { anos } \\
(2007-2017)\end{array}$ & 11 & 44 & 491 & $\begin{array}{c}28 \\
(5,7 \%)\end{array}$ \\
\hline Cadernos de Pesquisa & $\begin{array}{c}11 \text { anos } \\
(2007-2017)\end{array}$ & 11 & 37 & 478 & $\begin{array}{c}13 \\
(3,0 \%)\end{array}$ \\
\hline $\begin{array}{c}\text { Educational Administration } \\
\text { Quarterly }\end{array}$ & $\begin{array}{c}11 \text { anos } \\
(2007-2017)\end{array}$ & 11 & 55 & 260 & $\begin{array}{c}27 \\
(10,4 \%)\end{array}$ \\
\hline Jornal de Políticas Educacionais & $\begin{array}{c}11 \text { anos } \\
(2007-2017)\end{array}$ & 11 & 21 & 149 & $\begin{array}{c}15 \\
(10,6 \%)\end{array}$ \\
\hline $\begin{array}{l}\text { Revista Brasileira de Política e } \\
\text { Administração da Educação }\end{array}$ & $\begin{array}{c}11 \text { anos } \\
(2007-2017)\end{array}$ & 11 & 32 & 305 & $\begin{array}{c}29 \\
(9,8 \%)\end{array}$ \\
\hline $\begin{array}{c}\text { American Educational Research } \\
\text { Journal }\end{array}$ & $\begin{array}{c}11 \text { anos } \\
(2007-2017)\end{array}$ & 11 & 58 & 410 & $\begin{array}{c}27 \\
(6,6 \%)\end{array}$ \\
\hline TOTAL & -- & 76 & 283 & 2530 & $\begin{array}{c}153 \\
(6,1 \%)\end{array}$ \\
\hline
\end{tabular}

Fonte: Dados da pesquisa organizados pela autora.

O quadro acima destaca a relevância das publicações sobre o tema em relação ao volume de artigos publicados em cada periódico. Analisando a última coluna, percebe-se uma maior presença de artigos sobre a implementação de políticas educacionais nos periódicos de temática

2 AERA = American Educational Research Association; ANPAE = Associação Nacional de Política e Administração da Educação; ANPED = Associação Nacional de Pós-Graduação e Pesquisa em Educação; CEDES $=$ Centro de Estudos Educação e Sociedade; FCC $=$ Fundação Carlos Chagas; NUPE $=$ Núcleo de Políticas Educacionais; UCEA = University Council for Educational Administration. 
mais específica (Educational Administration Quarterly, Jornal de Políticas Educacionais e Revista Brasileira de Política e Administração da Educação) do que nos outros veículos cuja temática é mais ampla. Ainda assim, é preciso destacar uma baixa representatividade da temática no campo, uma vez que os artigos selecionados correspondem somente a 6,1\% do total de artigos publicados no período nas revistas selecionadas. Este destaque aponta tanto para a reflexão sobre a necessidade de ampliar os estudos sobre a implementação de políticas públicas educacionais quanto para a necessária discussão sobre o uso do conceito "implementação" no campo das políticas educacionais. Porém, aprofundar esta discussão foge ao escopo deste levantamento exploratório.

A partir da seleção dos 153 artigos, dedicamo-nos, em nosso grupo de pesquisa, à leitura detalhada de cada um deles. Metodologicamente, optamos por utilizar um modelo de fichamento que proporcionasse o registro das principais categorias que pretendíamos analisar. Os registros favoreceram a análise transversal dos artigos, levando-nos a aprofundar a discussão em cada etapa do estudo. Inicialmente, destacamos suas principais referências teóricas buscando identificar possíveis tendências na abordagem dos estudos. Em seguida, classificamos suas opções metodológicas especialmente considerando a presença de estudos teóricos ou empíricos em cada periódico e no volume geral de publicações. Finalmente, realizamos uma categorização temática das publicações. Os resultados destas análises são apresentados nas seções que se seguem.

\section{Estudos sobre implementação de políticas públicas educacionais: tendências teóricas e metodológicas}

Esta seção está dividida em duas partes, onde são apresentadas as tendências teóricas e metodológicas dos 153 artigos selecionados. Nosso objetivo foi identificar e analisar com base em que referências e a partir de quais metodologias os autores desenvolveram seus estudos sobre implementação de políticas públicas na educação básica.

\section{Tendências teóricas}

Um dos objetivos deste levantamento foi verificar quais eram as principais referências teóricas utilizadas nas pesquisas relatadas pelos artigos selecionados, ou seja, em se tratando de estudos sobre a implementação de políticas educacionais, quais autores aparecem como principais referências nas recentes pesquisas publicadas sobre o tema? Para responder esta questão, na leitura de cada artigo, listamos os principais autores citados na fundamentação e diálogo com as pesquisas.

Entre as recorrências no referencial teórico quantificadas, destacamos, nas publicações nacionais, os autores João Barroso (tipos de regulação e níveis de regulação), Weber (poder e dominação), Stephen Ball (ciclo de políticas), Bourdieu (representatividade social). Já entre os trabalhos internacionais há uma recorrência na citação dos estudos de Spillane (processos políticos intraescolares), Leithwood (liderança) e Lipsky (agentes implementadores das políticas públicas). Apresentamos, a seguir, alguns exemplos de como os autores dos estudos levantados operaram com os conceitos de regulação, agentes implementadores e discricionariedade em seus estudos sobre implementação de políticas públicas educacionais.

Barroso (2006) trabalha com o conceito de regulação para a análise de políticas educacionais considerando seu caráter institucional (governo e sua hierarquia) e seu caráter autônomo (atribuído aos atores). Em seus estudos são apresentadas três formas de regulação dessas políticas, as quais podem ser chamadas de: transnacional, nacional, e microrregulação local. 
Utilizando o termo "metarregulação", Barroso (2006) propõe romper com a ideia de verticalização do poder do estado na implementação das políticas educacionais, passando este ao papel de regulador das regulações. Krawczyk (2008), recorre ao conceito de regulação transnacional, a partir de Barroso (2006) para analisar as influências internacionais na implementação do Plano de Desenvolvimento da Educação - PDE no Brasil. Para Dourado (2007), o conceito de regulação é central para a análise dos limites e perspectivas das interfaces entre gestão e políticas públicas no Brasil. $\mathrm{O}$ autor inicia sua reflexão destacando a utilização do conceito, de acordo com Barroso (2006, p. 13), para "descrever dois tipos diferenciados de fenômeno, mas interdependentes: os modos como são produzidas e aplicadas as regras que orientam a ação dos atores; os modos como esses mesmos atores se apropriam delas e as transformam". A partir desta perspectiva, Dourado (2007) analisa as políticas e a gestão da educação básica no Brasil, especialmente destacando sua descontinuidade e centralização. No caso dos três programas analisados em seu estudo, o autor destaca a busca de equilíbrio entre centralização e descentralização, o que nos leva a refletir sobre o espaço de autonomia dos gestores educacionais no processo de reconstrução das políticas.

Ao analisar as características contextuais que poderiam influenciar a implementação de uma política específica para formação de professores alfabetizadores, Mangin (2009) se refere aos estudos de Lipsky ao argumentar que as reformas educacionais sofrem mudanças ou são subvertidas no nível escolar. $\mathrm{Na}$ conclusão de seu estudo, a autora destaca que o sucesso de uma política depende da habilidade de mobilizar as condições necessárias para sua implementação e esta é contextualmente determinada, como pôde comprovar nos 20 distritos estudados. Mayer e colegas (2013), por sua vez, analisam a implementação de uma política sobre a prática docente em diferentes contextos, com o foco nas características organizacionais que podem influenciar esta implementação. Fundamentando sua análise nos estudos dos burocratas de nível de rua (Lipsky, 1980), os autores concluem que a implementação da mesma política variou nas 6 escolas estudadas. E esta variação dependeu, principalmente, das seguintes variáveis testadas: relações entre a equipe da escola e o distrito, o apoio da direção da escola para as tomadas de decisão, o nível de confiança estabelecido entre os atores expresso na cultura escolar e o espaço de participação dos professores. Lançando luz ao espaço de discricionariedade dos sujeitos, o estudo especialmente destaca como as relações entre as pessoas (distrito/diretores/professores) influencia as diferentes formas de implementação da política. Koyama (2014), por sua vez, investiga as diferentes formas de atuação dos diretores frente às demandas de responsabilização da política No Child Left Behind. Destaca-se o importante papel dos diretores na implementação de decisões nacionais, especialmente negociando e aproximando-se às exigências externas, de maneiras às vezes inovadoras e às vezes conservadoras. A autora considera os diretores como policymakers locais, classificando-os como burocratas de nível de rua a partir da definição de Lipsky: "trabalhadores do serviço público encarregados da implementação de política púbica como parte de suas atividades diárias" (KOYAMA, 2014, p. 282, minha tradução). Acrescenta, ainda, a análise de Spillane e colegas (2002, apud KOYAMA, 2014, p. 282) que categoriza os diretores escolares como mediadores ou interlocutores entre o distrito central e as escolas, seletivamente negociando e mediando a implementação de políticas de responsabilização. Findlay (2015), que realiza um estudo em 10 escolas canadenses, também opera com o conceito de burocratas de nível de rua ao destacar o papel dos diretores escolares na implementação de políticas educacionais. Segundo o autor, os diretores entendem o seu espaço de discricionariedade como um desafio complexo, ao mesmo tempo como uma possibilidade de serem justos e adaptarem as regras.

O enfoque dado à referências teóricas dos artigos selecionados, mais do que quantificar citações pretendeu analisar como os autores lidos operavam com os conceitos considerados centrais para o estudo sobre a implementação das políticas educacionais. Os exemplos acima 
ilustram este processo que, além de ajudar-nos a refletir sobre nossa própria pesquisa, abre a discussão para o campo de produção científica nesta temática.

\section{Tendências metodológicas}

A leitura dos artigos selecionados neste levantamento permitiu, também, uma análise sobre os diferentes enfoques metodológicos adotados nestas pesquisas. A classificação que se segue, realizada a partir das estratégias metodológicas descritas nos artigos, tomou como referência a literatura específica da área sobre a trajetória da pesquisa qualitativa em educação (BOGDAN; BIKLEN, 1994); sobre as pesquisas quantitativas em educação no Brasil (GATTI, 2004) e sobre as relações comparativas estabelecidas na área educacional entre as metodologias de pesquisa qualitativas e quantitativas (GÜNTHER, 2006).

$\mathrm{Na}$ análise das opções metodológicas das pesquisas levantadas, os 153 artigos foram classificados em quatro grupos: a. Estudos teóricos; b. Pesquisas qualitativas; c. Pesquisas quantitativas; d. Pesquisas quanti-quali. $\mathrm{O}$ quadro a seguir sintetiza a distribuição dos artigos a partir desta seleção quanto à sua metodologia e os parágrafos que se seguem apresentam alguns exemplos dos artigos de cada grupo.

Quadro 2 - Levantamento Bibliográfico - Opções metodológicas

\begin{tabular}{|c|c|c|c|c|c|}
\hline Revistas & $\begin{array}{l}\text { Estudo } \\
\text { Teórico }\end{array}$ & $\begin{array}{l}\text { Pesquisa } \\
\text { Qualitativa }\end{array}$ & $\begin{array}{c}\text { Pesquisa } \\
\text { Quantitativa }\end{array}$ & $\begin{array}{c}\text { Pesquisa } \\
\text { Quanti-Quali }\end{array}$ & Total \\
\hline Cadernos de Pesquisa & 7 & 2 & 2 & 2 & 13 \\
\hline Educação e Sociedade & 26 & 1 & 1 & 0 & 28 \\
\hline Revista Brasileira de Educação & 7 & 3 & 4 & 0 & 14 \\
\hline $\begin{array}{l}\text { Jornal de Políticas } \\
\text { Educacionais }\end{array}$ & 6 & 6 & 1 & 2 & 15 \\
\hline $\begin{array}{l}\text { Revista Brasileira de Política e } \\
\text { Administração da Educação }\end{array}$ & 22 & 7 & 0 & 0 & 29 \\
\hline $\begin{array}{c}\text { Educational } \\
\text { Administration Quarterly }\end{array}$ & 3 & 14 & 3 & 7 & 27 \\
\hline $\begin{array}{l}\text { American Educational } \\
\text { Research Journal }\end{array}$ & 2 & 15 & 8 & 2 & 27 \\
\hline TOTAL & 73 & 48 & 19 & 13 & 153 \\
\hline
\end{tabular}

Fonte: Dados da pesquisa organizados pela autora.

Destacamos a predominância dos estudos teóricos (47\% no total), especialmente na RBPAE e na revista Educação e Sociedade. Entre estes estudos, destacam-se os temas Federalização, Financiamento, o Plano Nacional da Educação - PNE, o Plano de Desenvolvimento da Educação - PDE. O trabalho de Amaral (2017), por exemplo, traz uma análise documental sobre o orçamento da União e o investimento em educação e França (2014) se utiliza de indicadores educacionais e financeiros para analisar o regime de colaboração entre os entes federados no âmbito da implementação do PNE. Outros estudos, como o estudo de Welner (2012), Vieira (2007) e de Cohen e Mehta (2017) debruçam-se sobre a definição de conceitos no campo das políticas educacionais. Já Mainardes (2008), aponta as tendências dos estudos sobre a implementação da política de organização da escolaridade em ciclos no Brasil. Marques, Andrade e Azevedo (2017), por sua vez, fazem um denso estudo sobre as contribuições 
da análise do discurso para o estudo sobre a implementação de políticas públicas no campo educacional.

As pesquisas qualitativas, presentes em aproximadamente $32 \%$ dos estudos eram, em sua maioria, estudos de caso e poucos deles declararam o uso de softwares como o NVivo. Temponi e Machado (2011), por exemplo, analisam o Programa de Educação Integral Integrada, em Contagem/MG, a partir de análise documental e dos relatos dos seis coordenadores locais do programa entrevistados. A partir destas informações, os autores apontam desafios e avanços do programa, mas oferecem poucos detalhes sobre a metodologia empregada. O estudo de Silva (2017), que articula análise documental aos dados de questionários e entrevistas semiestruturadas realizados com os secretários municipais de educação de 12 municípios de Goiás, buscou analisar as limitações do Plano de Ações Articuladas (PAR) no cumprimento do propósito de fortalecer as relações de colaboração entre os municípios e a União. Também com a intenção de analisar a implementação da política educacional no nível meso, Duarte e Cardoso (2014) realizam 23 entrevistas com dirigentes municipais no Nordeste do Brasil. Os autores pretendiam analisar as lógicas de ação que orientam a atuação destes atores na regulação do sistema educacional brasileiro. Em uma perspectiva mais voltada para o nível micro de regulação das políticas educacionais, Botler (2015) realiza um estudo de caso de cunho etnográfico em uma escola pública de Recife. A autora analisa o conflito existente entre as políticas educacionais e sua implementação nas escolas, especialmente destacando a contradição entre autonomia e regulação. A metodologia do estudo incluiu observação intensiva, entrevistas e conversas informais com diferentes sujeitos da escola: diretor, coordenador pedagógico, professor, mãe de aluno.

As pesquisas quantitativas tiveram presença escassa entre os artigos levantados (aproximadamente 11\%) e algumas se limitaram à análise descritiva dos dados, como o estudo de Barretto (2015) sobre o trabalho docente e o artigo de Bauer e colegas (2017) sobre a implementação de políticas de avaliação em larga escala. Andrews e Vries (2012) utilizam dados relacionados ao Índice de Desenvolvimento da Educação Básica - IDEB para correlacionar níveis de pobreza e resultados educacionais em mais de 5.500 municípios brasileiros. Os resultados indicam a relação entre as variáveis e a relevância da implementação de políticas locais para favorecer diminuir o impacto das condições socioeconômicas na determinação dos níveis de aprendizagem sem, contudo, cair em políticas de culpabilização. Também buscando relacionar as políticas educacionais municipais ao desempenho dos alunos nas avaliações de larga escala, Alves (2008) realiza um estudo a partir dos dados educacionais de capitais brasileiras. Através da análise estatística a partir de modelos multiníveis, a autora indica as variáveis contextuais (características das políticas educacionais locais) relacionadas à variação do desempenho das escolas, apontando importantes indicações para a agenda da política educacional. Outros estudos quantitativos, especialmente aqueles encontrados nas revistas internacionais, fizeram uso de modelagens estatísticas para estimar relações entre variáveis como: a implementação de investimento na melhoria das escolas em diferentes estados (DAVIS, 2015); a interação entre diretores e agentes do distrito na implementação das políticas educacionais (LIOU, 2016); a percepção dos professores sobre aspectos escolares ao longo da implementação de uma política (HECK; CHANG, 2017); entre outras.

Entre os poucos trabalhos que propuseram uma abordagem metodológica mista, destacamos o estudo de Érnica e Batista (2012), em São Paulo, que envolveu a elaboração do Índice de Vulnerabilidade Social para ser relacionado estatisticamente ao desempenho médio das escolas estudadas. Os autores agregam à análise dados de pesquisa qualitativa (entrevistas e observação) realizada em uma amostra de escolas daquele espaço. Já Costa e Koslinski (2011), no Rio de Janeiro, utilizam regressões logísticas com os dados da secretaria de educação municipal daquela cidade e mais dados coletados num survey aplicado a alunos na mesma rede para estimar a 
probabilidade de diferentes perfis de alunos estarem matriculados em escolas consideradas "de prestigio". Dados qualitativos coletados em entrevistas e observação em algumas unidades da amostra complementam a análise dos autores sobre os processos previstos na política de matrículas nas escolas desta rede, denunciando diferentes formas de adaptação destes processos. Em um olhar mais voltado para a implementação de políticas no contexto da relação entre o nível intermediário (Coordenadorias Regionais de Educação - CREs) e as unidades escolares, Carrasqueira e colegas (2015) também desenvolvem um estudo no Rio de Janeiro. Utilizam dados estatísticos para classificar as CREs em mais ou menos equitativas a partir do desvio padrão entre os resultados das escolas. Os dados qualitativos, coletados a partir de entrevistas com as coordenadoras das CREs que atuam diretamente no contato com as escolas, completam a análise dos autores sobre as diferentes estratégias adotadas pelas CREs estudadas. Matsumura e colegas (2009), por sua vez, utilizam dados de survey e entrevistas para estimar as relações entre a liderança do diretor e a variação no nível de adesão dos professores a um programa de formação para a alfabetização no contexto dos Estados Unidos.

Como pôde ser observado na classificação apresentada no quadro 3, entre as metodologias empregadas para os estudos empíricos, destacam-se as estratégias de cunho qualitativo. Porém, mais do que quantificar as opções metodológicas dos artigos lidos e analisados neste levantamento, pretendeu-se trazer elementos destas pesquisas que favoreçam a reflexão sobre os caminhos que podem ser trilhados na proposição de pesquisas sobre a implementação de políticas públicas na área educacional.

\section{Estudos sobre implementação de políticas públicas educacionais: categorização temática}

A partir da leitura completa dos 153 artigos selecionados, buscamos identificar suas tendências temáticas especialmente considerando a apresentação dos estudos, suas questões de pesquisa (no caso dos estudos empíricos), suas análises e considerações. Para cada artigo foi designada uma ou duas palavras-chave que, em posterior análise foram agrupadas dependendo de sua proximidade. Assim, os artigos foram finalmente categorizados em 5 grandes grupos, apresentados a seguir:

Gráfico 1 - Levantamento Bibliográfico - Categorização temática

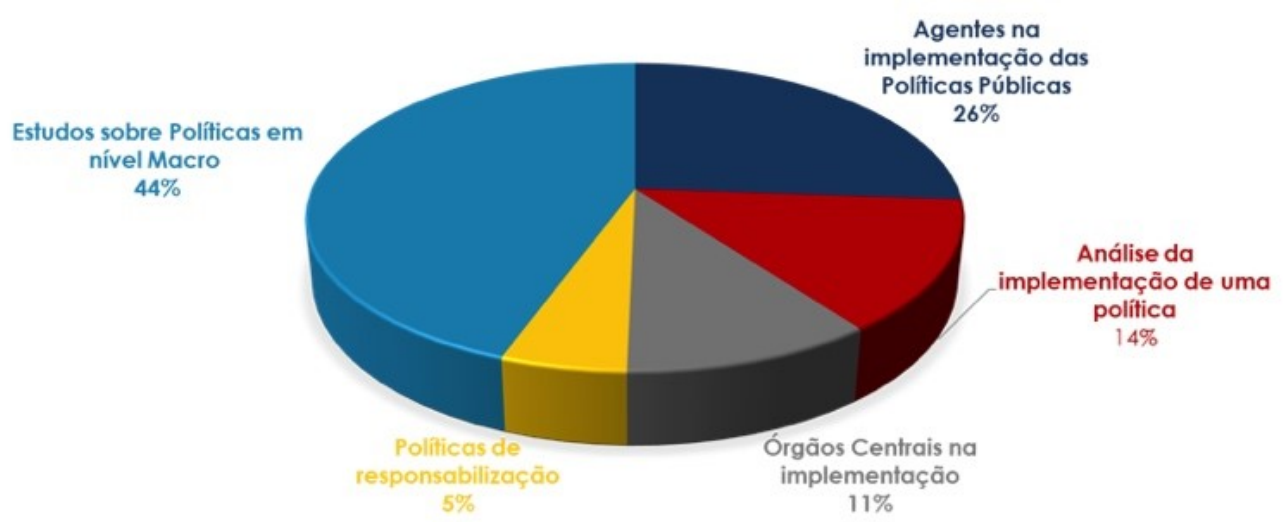

Fonte: Produzido pelo grupo de pesquisa.

Como pode ser visto no gráfico, há uma predominância de artigos que realizaram estudos sobre políticas educacionais em um nível macro, em sua maioria analisando o histórico e o contexto da implementação de uma nova política a partir da análise documental ou do histórico da formulação da política. Neste grupo encontram-se prioritariamente os estudos teóricos sobre 
o PNE, o PDE, o PAR, sobre Federalismo e municipalização, sobre as políticas de financiamento (CURY, 2007) ou sobre as rupturas e permanências nas políticas educacionais (OLIVEIRA, 2009). Em comum, todos eles se voltavam mais para os processos de agenda e formulação das políticas públicas educacionais.

Os artigos que enfocaram as políticas de responsabilização, em menor quantidade, apresentavam análises sobre as mudanças trazidas pela introdução de tais políticas (MEHTA, 2013), como por exemplo as consequências para os professores (HARRIS et al., 2014), estando mais em uma linha de estudos sobre a avaliação das políticas.

As três últimas categorias, à direita do gráfico, contemplam os estudos que mais se voltaram para uma análise da implementação das políticas, especialmente destacando o papel dos agentes neste processo. Entre aqueles que estudaram o papel dos órgãos centrais, destacamos a pesquisa sobre o papel da Secretaria Municipal de Educação - SME e das CREs nas políticas educacionais do Rio de Janeiro (CARRASQUEIRA et al., 2015), e o trabalho de Slavin e colegas (2013) que enfocou o uso de dados pelos órgãos centrais para acompanhar a implementação das políticas educacionais.

Entre os estudos que se dedicaram a analisar a implementação de uma política em um contexto específico, temos aqueles que analisaram as políticas de avaliação em larga escala em capitais brasileiras (ALVES, 2008) e a implementação da política de educação em tempo integral de São Paulo (PARENTE, 2017). Entre as que se referiam a leis específicas dos Estados Unidos, destacam-se o trabalho de Brown e Clift (2010) que analisam a implementação do Adequate Yearly Progress e o estudo de Correnti e Rowan (2007) sobre implementação de três programas: Accelerated Schools Project (ASP), America's Choice (AC), e Success for All (SFA) em uma amostra de escolas.

E, em um último grupo, foram categorizados os trabalhos que abordaram o papel dos sujeitos na implementação das políticas públicas. Nestes estudos, em sua maioria trabalhos qualitativos, destaca-se o papel dos diferentes agentes (coordenadores distritais, diretores, professores etc.) na implementação de uma política educacional. Alguns deles abordam questões relacionadas à autonomia e discricionariedade destes atores no contexto escolar e suas possíveis consequências. Entre os trabalhos categorizados neste grupo, destaco: o artigo de Li (2017) que apresenta os processos de adaptação e novas práticas experimentados pelos educadores na implementação de uma nova política educacional; o estudo de Oliveira (2013) que analisa o papel da equipe de gestão na mediação de uma política educacional no Rio de Janeiro; o estudo de Mayer e colegas (2013) que mostrou como a implementação de uma mesma política em 6 escolas diferentes variou dependendo das ações e relações dos agentes; o trabalho de Craig (2009) que destacou o espaço de discricionariedade da sala de aula; o estudo de Rorrer et al (2008) que define categorias, a partir dos achados de pesquisas, sobre a atuação dos agentes dos distritos educacionais na reforma educacional em prol da qualidade da educação; o trabalho de Holme e Rangel (2012) que destaca a importância do contexto para as diferentes respostas à políticas educacionais; o trabalho de Anderson (2017) sobre a fidelidade dos agentes ao texto da política na implementação; a atenção dada por Placco, Souza e Almeida (2012) ao importante papel do coordenador pedagógico na implementação de políticas educacionais para a melhora da aprendizagem; o destaque dado por Koyama (2014) para o papel dos diretores na implementação das políticas educacionais. Pode-se considerar que, em geral, os trabalhos categorizados neste grupo e exemplificados acima trazem importantes contribuições para a reflexão sobre a implementação das políticas públicas educacionais a partir da análise do espaço de discricionariedade dos atores envolvidos, especialmente no nível meso e local de regulação. 
Como é esperado em um trabalho de revisão bibliográfica, a categorização dos artigos aqui proposta tomou como referência os estudos e leituras sobre o tema no âmbito do grupo de pesquisa no qual foi desenvolvida. Sendo assim, não pretende ser uma classificação definitiva, mas sim apresentar uma possível forma de interlocução com a produção neste campo.

\section{Considerações finais}

O presente artigo apresentou o resultado de um levantamento sobre as recentes publicações no campo da implementação das políticas educacionais, destacando seu aporte teórico e metodológico e, especialmente, propondo categorias de análise sobre as tendências de suas abordagens temáticas. As contribuições de uma revisão sistemática como a que foi proposta consistem tanto na aproximação à produção acadêmica acerca de um tema como na possibilidade de identificar suas tendências e categorias temáticas, ampliando as possibilidades de diálogo no campo. No caso deste levantamento, destaca-se: a) a ainda escassa presença de artigos sobre a implementação de políticas educacionais em importantes periódicos nacionais e internacionais correspondendo a 6,1\% das publicações nos periódicos analisados, entre 2007 e 2017; b) entre os artigos que abordavam a implementação de políticas públicas educacionais, um número expressivo de estudos teóricos em relação aos estudos empíricos; c) a presença de estudos que se dedicaram a analisar o papel dos agentes implementadores, utilizando conceitos (burocratas de nível de rua; discricionariedade; regulação) e referenciais teóricos (Barroso, Lipsky, Spillane) relevantes para os estudos desenvolvidos pelos grupos de pesquisa do qual este trabalho faz parte; d) a ausência de trabalhos que analisem, no mesmo estudo, o papel dos agentes implementadores em diferentes contextos (macro, meso e micro) de implementação das políticas educacionais.

Assim, apontando especificidades da produção acadêmica sobre o tema na última década, este trabalho pretende colaborar com as pesquisas no campo indicando possíveis agendas de pesquisa emergentes desta análise.

\section{Referências}

ALVES, F. Políticas educacionais e desempenho escolar nas capitais brasileiras. Cadernos de Pesquisa, São Paulo, v. 38, n. 134, p. 413-440, maio/ago. 2008. DOI: https://doi.org/10.1590/s0100-15742008000200008

AMARAL, N. C. Com a PEC 241/55 (EC 95) haverá prioridade para cumprir as metas do PNE (2014-2024)? Revista Brasileira de Educação, Rio de Janeiro, v. 22, n. 71, p. 1- 25, 2017. DOI: https://doi.org/10.1590/s1413-24782017227145

ANDERSON, E. R. Accommodating change: relating fidelity of implementation to Program Fit in Educational Reforms. American Educational Research Journal, v. 54, n. 6, p. 1288-1315, 2017. DOI: https://doi.org/10.3102/0002831217718164

ANDREWS, C.; VRIES, M. Pobreza e municipalização da educação: Análise dos resultados do IDEB (2005-2009). Cadernos de pesquisa, São Paulo, v. 42, n. 147, p. 826-847, set./dez. 2012. DOI: https://doi.org/10.1590/s0100-15742012000300010

BARRETTO, E. S. S. Políticas de formação docente para a educação básica no Brasil: embates contemporâneos. Revista Brasileira de Educação, Rio de Janeiro, v. 20, n. 62, p. 679-701, jul./set. 2015. DOI: https://doi.org/10.1590/s1413-24782015206207 
BARROSO, J. (Org.) A regulação das políticas públicas de educação: espaços, dinâmicas e atores. Lisboa: Educa, 2006.

BAUER, A. et al. Iniciativas de avaliação do ensino fundamental em municípios brasileiros: mapeamento e tendências. Revista Brasileira de Educação, Rio de Janeiro, v. 22, n. 71, p. 1-19, 2017. DOI: $\underline{\text { https://doi.org/10.1590/s1413-24782017227153 }}$

BOGDAN, R.; BIKLEN, S. Investigação qualitativa em educação. Porto: Porto Editora, 1994.

BOTLER, A. M. H. Repercussões das políticas educacionais na organização escolar: o fator tempo entre a autonomia e a regulação. Revista Brasileira de Política e Administração da Educação, Goiânia, v. 31, n. 1, p. 107-124, jan./abr. 2015. DOI: https://doi.org/10.21573/vol31n12015.58919

BROWN, A. B.; CLIFT, J. W. The unequal effect of adequate yearly progress: evidence from school visits. American Educational Research Journal, v. 47, n. 4, p. 774-798, 2010. DOI: https://doi.org/10.3102/0002831210374644

CARRASQUEIRA, K. et al. Políticas de responsabilização escolar: um estudo exploratório sobre as estratégias de instâncias intermediárias na rede municipal do Rio de Janeiro. Jornal de Políticas Educacionais, Curitiba, v. 9, n. 17-18, p. 75-89, 2015. DOI: https://doi.org/10.5380/ipe.v9i17/18.41299

COHEN, D. K.; MEHTA, J. D. Why reform sometimes succeeds: understanding the conditions that produce reforms that last. American Educational Research Journal, v. 54, n. 4, p. 644690, apr. 2017. DOI: https://doi.org/10.3102/0002831217700078

CORRENTI, R.; ROWAN, B. Opening up the black box: literacy instruction in schools participating in three comprehensive school reform programs. American Educational Research Journal, v. 44, n. 2, p. 298-339, jun. 2007. DOI: https://doi.org/10.3102/0002831207302501

COSTA, M.; KOSLINSKI, M. C. Quase-mercado oculto: disputa por escolas "comuns" no Rio de Janeiro. Cadernos de Pesquisa, São Paulo, v. 41, n. 142, p. 246-266, jan./abr. 2011. DOI: https://doi.org/10.1590/s0100-15742011000100013

CRAIG, C. J. The contested classroom space: a decade of lived educational policy in Texas schools. American Educational Research Journal, v. 46, n. 4, p. 1034-1059, dec. 2009. DOI: https://doi.org/10.3102/0002831209334843

CURY, C. R. J. Estado e políticas de financiamento em educação. Educação \& Sociedade, Campinas, v. 28, n. 100, p. 831-855, out. 2007. DOI: https://doi.org/10.1590/s0101$\underline{73302007000300010}$

DAVIES, P. Revisões sistemáticas e a Campbell Collaboration. In: THOMAS, G.; PRING, R. (Org.). Educação baseada em evidências: a utilização dos achados científicos para a qualificação da prática pedagógica. Porto Alegre: Artmed, 2007. p. 31-43.

DAVIS, T. E. State and Federal Policies for school facility construction - a comparison of Michigan and Ohio. Educational Administration Quarterly, v. 51, n. 1, p. 3-26, nov. 2015. DOI: https://doi.org/10.1177/0013161x13508773 
DOURADO, L. F. Políticas e gestão da educação básica no Brasil: limites e perspectivas. Educação \& Sociedade, Campinas, v. 28, n. 100, p. 921-946, out. 2007. DOI: https://doi.org/10.1590/s0101-73302007000300014

DUARTE, M.; CARDOSO, M. Dirigentes municipais de educação no Brasil: regulação intermediária do sistema educacional. Revista Brasileira de Política e Administração da Educação, Goiânia, v. 30, n. 3, p. 513-533 set./dez. 2014.

ÉRNICA, M.; BATISTA, A. A. G. A escola, a metrópole e a vizinhança vulnerável. Cadernos de Pesquisa, São Paulo, v. 42, n. 146, p. 640-666, maio/ago. 2012. DOI: https://doi.org/10.1590/s0100-15742012000200016

FINDLAY, N. M. Discretion in student discipline - insight into elementary principals' decision making. Educational Administration Quarterly, v. 51, n. 3, p. 472-507, may 2015. DOI: https://doi.org/10.1177/0013161x14523617

FRANÇA, M. Plano Nacional de Educação e o regime de colaboração: os indicadores educacionais e financeiros. Revista Brasileira de Política e Administração da Educação, Goiânia, v. 30, n. 2, p. 417-433, maio/ago. 2014.

GATTI, B. A. Estudos quantitativos em educação. Educação e Pesquisa, São Paulo, v. 30, n. 1, p. 11-30, jan./abr. 2004. DOI: https://doi.org/10.1590/s1517-97022004000100002

GÜNTHER, H. Pesquisa qualitativa versus Pesquisa quantitativa: esta é a questão? Psicologia: Teoria e Pesquisa, Brasília, v. 22, n. 2, p. 201-210, maio/ago. 2006. DOI: https://doi.org/10.1590/s0102-37722006000200010

HARRIS, D. N.; INGLE, W. K.; RUTLEDGE, S. A. How teacher evaluation methods matter for accountability: a comparative analysis of teacher effectiveness ratings by principals and teacher value-added measures. American Educational Research Journal, v. 51, n. 1, p. 73-112, feb. 2014. DOI: https://doi.org/10.3102/0002831213517130

HECK, R. H.; CHANG, J. Examining the timing of educational changes among elementary schools after the implementation of NCLB. Educational Administration Quarterly, v. 53, n. 4, p. 649-694, jun. 2017. DOI: https://doi.org/10.1177/0013161x17711480

HOLME, J. J.; RANGEL, V. S. Putting school reform in its place: social geography, organizational social capital, and school performance. American Educational Research Journal, v. 49, n. 2, p. 257-283, apr. 2012. DOI: https://doi.org/10.3102/0002831211423316

KOYAMA, J. Principals as Bricoleurs - Making Sense and Making Do in an Era of accountability. Educational Administration Quarterly, v. 50, n. 2, p. 279-304, jul. 2014. DOI: https://doi.org/10.1177/0013161x13492796

KRAWCZYK, N. R. O PDE: novo modo de regulação estatal? Cadernos de Pesquisa, São Paulo, v. 38, n. 135, p. 797-815, set./dez. 2008. DOI: https://doi.org/10.1590/s0100$\underline{15742008000300013}$

LIOU, Y. Tied to the common core - exploring the characteristics of reform advice relationships of educational leaders. Educational Administration Quarterly, v. 52, n. 5, p. 793-840, ago. 2016. DOI: $\underline{\text { https://doi.org/10.1177/0013161x16664116 }}$

LIPSKY, M. Street-level bureaucracy: dilemmas of the individual in public service. New York: Russel Sage Foundation, 1980. 
LOTTA, G. S. Burocracia e Implementação de Políticas de Saúde: os agentes comunitários na Estratégia Saúde da Família. Rio de Janeiro: Editora Fiocruz, 2015.

MAINARDES, J. A organização da escolaridade em ciclos no Brasil: uma análise de pesquisas sobre processos de implementação. Revista Brasileira de Política e Administração da Educação, Goiânia, n. 1, v. 24, p. 11-30, jan./abr. 2008.

MAINARDES, J.; STREMEL, S. A teoria de Basil Bernstein e algumas de suas contribuições para as pesquisas sobre políticas educacionais e curriculares. Teias, Rio de Janeiro, v. 11, n. 22, p. 31-54, maio/ago. 2010.

MANGIN, M. M. Literacy Coach role implementation: how district context influences reform efforts. Educational Administration Quarterly, v. 45, n. 5, p. 759-792, out. 2009. DOI: https://doi.org/10.1177/0013161x09347731

MARQUES, L. R.; ANDRADE, E. F.; AZEVEDO, J. M. Pesquisa em política educacional e discurso: sugestões analíticas. Revista Brasileira de Política e Administração da Educação. Goiânia, v. 33, n. 1, p. 55-71, jan./abr. 2017. DOI: https://doi.org/10.21573/vol33n12017.72834

MATSUMURA, L. C. et al. Leadership for literacy coaching: the principal's role in launching a new coaching program. Educational Administration Quarterly, v. 45, n. 5, p. 655-693, oct. 2009. DOI: https://doi.org/10.1177/0013161x09347341

MAYER, A. M. et al. Negotiating site-based management and expanded teacher decision making - a case study of six urban schools. Educational Administration Quarterly, v. 49, n.5, p. 3-51, jul. 2013. DOI: https://doi.org/10.1177/0013161x13492793

MEHTA, J. How Paradigms Create Politics: The Transformation of American Educational Policy, 1980-2001. American Educational Research Journal, v. 50, n. 2, p. 285-324, apr. 2013. DOI: https://doi.org/10.3102/0002831212471417

OLIVEIRA, D. As políticas educacionais no governo Lula: rupturas e permanências. Revista Brasileira de Política e Administração da Educação, Goiânia, v. 25, n. 2, p. 197-209, maio/ago. 2009.

OLIVEIRA, A. C. P. Política pública e gestão escolar: um estudo de caso no Rio de Janeiro. Jornal de Políticas Educacionais, Curitiba, v. 7, n. 14, p. 29-37, jul./dez. 2013. DOI: https://doi.org/10.5380/jpe.v7i14.31530

PARENTE, C. M. D. Políticas de educação integral em tempo integral no estado de São Paulo: modelos e experiências. Jornal de Políticas Educacionais, Curitiba, v. 11, n. 24, p. 11-23, dez. 2017. DOI: https://doi.org/10.5380/ipe.v1110.55513

PLACCO, V. M. N. de S.; SOUZA, V. L. T. de; ALMEIDA, L. R. de. O coordenador pedagógico: aportes à proposição de políticas públicas. Cadernos de Pesquisa, São Paulo, v. 42, n. 147, p. 754-771, set./dez. 2012. DOI: https://doi.org/10.1590/s0100-15742012000300006

RORRER, A. K.; SKRLA, L.; SCHEURICH, J. J. Districts as Institutional Actors in Educational Reform. Educational Administration Quarterly, v. 44, n. 3, p. 307-358, aug. 2008. DOI: https://doi.org/10.1177/0013161x08318962

SILVA, L. G. A. O Plano de Ações Articuladas e o regime de colaboração: promessas não cumpridas de fortalecimento das relações de colaboração entre os municípios e a união. Revista 
Brasileira de Política e Administração da Educação, Goiânia, v. 33, n. 2, p. 337-354, maio/ago. 2017. DOI: https://doi.org/10.21573/vol33n22017.70900

SLAVIN, R. E. et al. Effects of a data-driven district reform model on state assessment outcomes. American Educational Research Journal, v. 50, n. 2, p. 371-396, apr. 2013. DOI: https://doi.org/10.3102/0002831212466909

TEMPONI, F.; MACHADO, L. Políticas de educação em tempo integral, de intersetorialidade e de desenvolvimento local: um diálogo possível? Revista Brasileira de Política e Administração da Educação, Goiânia, v. 27, n. 3, p. 361-588, set./dez. 2011.

VIEIRA, S. Políticas e gestão da educação básica: revisitando conceitos simples. Revista Brasileira de Política e Administração da Educação, Goiânia, v. 23, n. 1, p. 53-69, jan./abr. 2007.

WELNER, K. G. Scholars as Policy Actors: Research, Public Discourse, and the Zone of Judicial Constraints. American Educational Research Journal, v. 49, n. 1, p. 7-29, feb. 2012. DOI: https://doi.org/10.3102/0002831211415253

Recebido em 16/11/2018

Versão corrigida recebida em 18/03/2019

Aceito em 08/04/2019

Publicado online em 12/04/2019

\section{Ana Cristina Prado de Oliveira}

Professora Adjunta da Universidade Federal do Estado do Rio de Janeiro - UNIRIO. Departamento de Fundamentos da Educação 